There were no serious treatment emergent adverse events (TEAEs). 15 patients (19\%) randomised to MBS2320 withdrew due to TEAEs, predominantly of nausea. TEAEs were typically reported soon after dosing, were mostly mild in severity and resolved without treatment. Onset of TEAEs reduced as the study proceeded.

Gastrointestinal disorders were the most frequently reported TEAEs (all causalities) with a higher incidence in patients receiving MBS2320 (68.8\%) than placebo (14.6\%). Nausea was most frequently reported during Week 1 (27.3\% patients). Asthenia and/or fatigue was reported more frequently in the MBS2320 treatment group $(23.8 \%$ patients) than with placebo $(7.3 \%$ patients), with the majority being considered related to study drug. Infections were more frequently reported by patients receiving placebo $(22.0 \%)$ than those receiving MBS2320 $(12.5 \%)$. There were no clinically relevant treatment-related trends in the biochemistry, haematology, urinalysis, vital signs or ECG data.

Higher ACR20 response rates were observed in patients receiving MBS2320 versus those receiving placebo at all time points and increased with time. At Week 12, ACR50 response rates with MBS2320 treatment were increased by $>4$-fold compared with placebo ( $11.6 \%$ vs $2.5 \%)$. Greater mean reductions from baseline in DAS28-CRP were also observed in patients receiving MBS2320 versus those receiving placebo at Week $12(-18.6 \%$ vs $-8.4 \%)$. DAS28-CRP responder rates were more than doubled with MBS2320 treatment compared to placebo (5\% vs $14 \%)$. These changes were mirrored by improvements in tender joint counts, reduced hsCRP and improvements in Patient Reported Outcomes of pain VAS, Patients' and Clinicians' Global Assessments of Disease Activity and Patients' Global Impression of Change.

Conclusion: MBS2320 was generally well tolerated for up to 12 weeks in this RA study population. Nausea was the most common TEAE, was generally mild in severity and resolved without treatment. In this population of patients with hardto-treat, severe, active, erosive disease MBS2320 showed evidence of a clinical benefit on both ACR20 responses and DAS28-CRP.

References:

[1] Patel et al, Ann Rheum Dis, 78, S2, 2019, A228

Acknowledgments: Louise Jopling, Ian Anderson, Ian Gourley, Devenini Damayanthi

Disclosure of Interests: Lisa Patel Shareholder of: Istesso Ltd, Employee of: Istesso Ltd, Laurence Skillern Consultant of: Istesso Ltd, Martyn Foster Shareholder of: Istesso Ltd, Consultant of: Istesso Ltd, Andy Gray Shareholder of: Istesso Ltd, Consultant of: Istesso Ltd, Richard Leff Consultant of: Istesso Ltd, Sam Williams Shareholder of: Istesso Ltd, Employee of: Istesso Ltd

DOI: 10.1136/annrheumdis-2020-eular.3804

\section{OP0235 ACHIEVING A LOW DAS IN THE FIRST 4-MONTHS AFTER DIAGNOSIS IS IMPORTANT FOR THE LONG TERM CHANCE OF ACHIEVING DMARD-FREE REMISSION}

M. Verstappen ${ }^{1}$, E. Niemantsverdriet ${ }^{1}$, A. Van der Helm - van Mil ${ }^{1,2}{ }^{1}{ }^{1}$ Leiden University Medical Center, Rheumatology, Leiden, Netherlands; ${ }^{2}$ Erasmus University Medical Center, Rheumatology, Rotterdam, Netherlands

Background: Sustained DMARD-free remission (SDFR) is increasingly achievable in RA. The pathogenesis underlying SDFR-development is unknown and patient-characteristics at diagnosis poorly explain whether SDFR will be achieved. This limits substantiated decisions to discontinue DMARD-treatment in clinical practice.

Objectives: To increase the understanding of SDFR, we studied the course of disease activity scores (DAS) over time in relation to SDFR-development. Subsequently, we explored whether DAS-time course could be helpful to identify patients likely to achieve SDFR.

Methods: 761 RA-patients consecutively included in the Leiden Early Arthritis Clinic, treated with initial methotrexate and treat-to-target treatment, were studied (median follow-up 7 years). The course of DAS was compared between patients achieving SDFR within 7 years and those who did not, using linear mixed models, stratified for ACPA. Subsequently, the relation between DAS at 4 months and the probability of achieving SDFR within 7 years was studied with logistic regression. Kaplan-Meier curves were constructed to illustrate cumulative incidence of SDFR for different DAS categories at 4 months, respectively $<1.6,1.6-2.4$, 2.4-3.6, >3.6.

Results: Patients achieving SDFR were characterized by a remarkedly different DAS response within 4 months after diagnosis. Compared to patients who did not achieve SDFR, the SDFR-group showed a prominently stronger decline in DAS between baseline and 4 months; 1.59 units decline $(95 \% \mathrm{Cl}, 1.24-1.95)$ versus 0.96 units $(95 \% \mathrm{Cl}, 0.85-1.07)$ decline $(\mathrm{p}<0.001)$ (figure 1$)$. Stratification for ACPA yielded a similar and statistically significant effect in ACPA-negative RA. In ACPA-positive RA this effect was absent. Subsequently, the probability of achieving SDFR during 7 years was studied in ACPA-negative RA and it was observed to be lower for patients with higher DAS at 4 months. After 7 years of disease, the cumulative incidence for SDFR in ACPA-negative patients with DAS $<1.6$ at 4 months was high $(71.0 \%)$, whilst SDFR was rare among those with DAS $>3.6$ at 4 months $(7.1 \%)$ (figure 2$)$.

Conclusion: In RA-patients treated according to current guidelines, SDFR is predominantly achieved in patients with a strong decline in DAS during the first four months after diagnosis. Especially in ACPA-negative RA, the DAS at 4-months can be useful for later decisions to stop DMARDs.
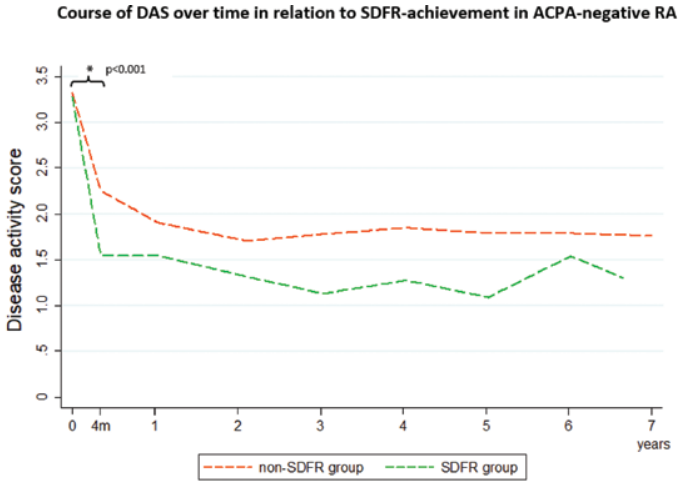

Figure 1. Course of disease activity scores over time in relation to SDFR-achievement in 7 years of follow-up in ACPA-negative RA. Course of disease activity scores (DAS) over time between ACPA-negative patients achieving SDFR (SDFR group) and those not achieving SDFR (non-SDFR group). " indicates significant stronger decline in DAS in SDFR-group. ACPA: anti-citrullinated protein antibodies, SDFR: sustained DMARD-free remission.

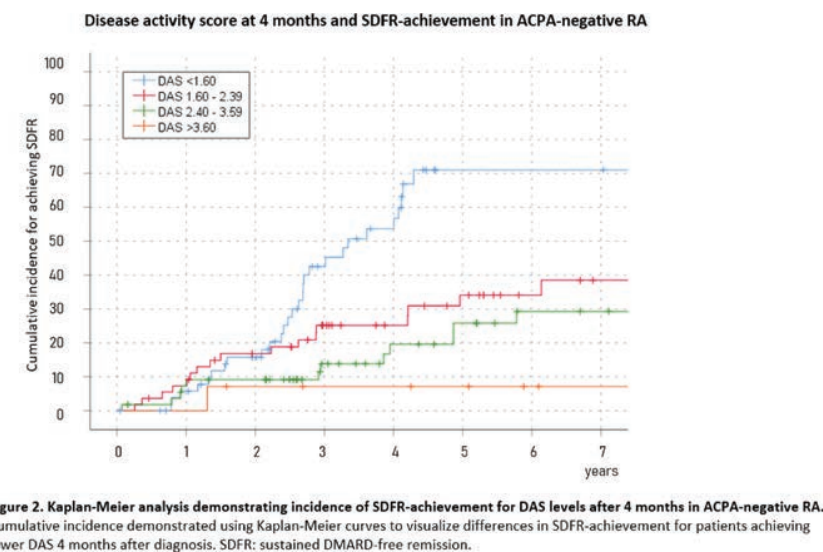

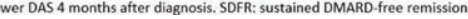

Disclosure of Interests: None declared

DOI: 10.1136/annrheumdis-2020-eular.1837

\section{OP0236 RELEVANCE OF BIASED PAR2 INHIBITORS IN REDUCING INFLAMMATION AND CARTILAGE DEGRADATION IN IN VITRO AND IN VIVO MODELS OF RHEUMATOID ARTHRITIS}

T. Brugat ${ }^{1}$, B. Rugeri ${ }^{1}$, G. Hommet ${ }^{1}$, A. Dumont ${ }^{1}$, L. Baron ${ }^{1}$, C. Halter ${ }^{1}$ M. Sémache ${ }^{2}$, A. Mancini ${ }^{2}$, C. Amalric ${ }^{1}$, M. Giambelluco ${ }^{1}$, N. Lenne ${ }^{1}$, M. Sidhoum ${ }^{1}$, C. Franchet ${ }^{1}$, S. Mayer ${ }^{1}$, X. Leroy ${ }^{1}$, S. Schann ${ }^{1} .{ }^{1}$ Domain Therapeutics, Illkirch, France; ${ }^{2}$ Domain Therapeutics, Montréal, Canada

Background: Protease-activated receptor-2 (PAR2) is a member of a family of G-protein-coupled receptors involved in multiple physiological mechanisms Compelling evidences have unravelled the key roles of PAR2 in the pathology of both rheumatoid arthritis (RA) and osteoarthritis $(O A)^{1}$. Indeed, in vitro, in vivo and ex vivo experiments showed that this receptor promotes inflammation, cartilage erosion (and subsequent bone degradation), and pain. However, the signalling pathways involved in these functions are not well understood ${ }^{2}$. This is of importance as some pathways can promote the pathogenesis ${ }^{3}$ while others prevent it ${ }^{4}$. We developed a new series of small molecules as novel biased PAR2 inhibitors to treat rheumatic diseases.

Objectives: To evaluate the efficacy and mechanism of action of new biased PAR2 inhibitors on cartilage erosion and inflammation.

Methods: The potency of compounds to inhibit human PAR2 signalling was evaluated in vitro by FLIPR calcium assay in HEK293 cells. The same assay was used to determine their selectivity over human PAR1 and PAR4 as well as murine versions of PAR2. The effect of several PAR2 inhibitors on 9 signalling pathways (Gi2, GoB, Gz, Gq, G13, G14, G15, B arrestin 2, EPAC) was evaluated by the BRET-based bioSens-AllTM technology. In vitro anti-hypertrophic effect was determined by measuring the mRNA level of type II collagen, aggrecan 
and MMP13 in rat chondrocytes after IL1 $\beta$ stimulation. In vitro anti-inflammatory effect was determined by measuring the secretion of IL6, IL8, IL1 $\beta$, TNFa and IFN $\gamma$ by human monocytes. In vivo, the pharmacodynamic of our small molecules was assessed after intravenous and oral administration. Therapeutic efficacy of a compound was then evaluated in a collagen-induced arthritis model in DBA $1 / \mathrm{J}$ mice. In this model, measures of the arthritis index score, body weight, plasma level of TNFa, IL6, IL8 and IL1 $\beta$ and histological evaluation of cartilage erosion were performed.

Results: Our new series of small molecules are potent PAR2 inhibitors (IC50<1nM in calcium assay) with some selectivity over PAR1 and PAR4. Our compounds significantly inhibited PAR2 mediated recruitment of $\mathrm{Gz}, \mathrm{Gq}, \mathrm{G} 13$, G14 and G15. However, surprisingly, these small molecules had no effect on B arrestin 2, EPAC, Gi2 and GoB demonstrating that they are biased inhibitors. The effect of our compounds on PAR2 signalling was clearly different from 3 already existing PAR2 inhibitors described in the literature (I-117, AZ3451 and P2pal-18s). We compared the in vitro anti-hypertrophic effect on chondrocyte and anti-inflammatory effect on monocytes of these compounds to determine the importance of PAR2 signalling pathways in these cellular functions. In vivo, our small molecules had good bioavailability after oral administration of $10 \mathrm{mg} / \mathrm{kg}$ in mice (clearance $=$ $0.038 \mathrm{~L} / \mathrm{h} / \mathrm{kg} ; \mathrm{T} 1 \frac{1}{2}=9.9 \mathrm{~h} ; \mathrm{AUC}=162564 \mathrm{ng} \cdot \mathrm{h} / \mathrm{mL} ; \mathrm{Cmax}=9005 \mathrm{ng} / \mathrm{mL}$ ). The in vivo therapeutic efficacy of a biased PAR2 inhibitor in a model of collagen-induced arthritis will be presented.

Conclusion: Our results show the potency of biased PAR2 inhibitors to reduce both the inflammation and cartilage erosion in rheumatoid arthritis. They confirm the huge potential of PAR2 as a therapeutic target and unravel the relevance of biased antagonism of this receptor to treat rheumatic diseases.

References:

[1] McCulloch et al., Frontiers in Endocrinology, 2018; ${ }^{2}$ Hollenberg et al., British

Journal of Pharmacology, 2014; ${ }^{3}$ Sharma et al., Genes and Immunity, 2015;

${ }^{4}$ Rayees et al., Cell Reports, 2019

Disclosure of Interests: Thibaut Brugat Employee of: Domain Therapeutics, Baptiste Rugeri Employee of: Domain Therapeutics, Gaël Hommet Employee of: Domain Therapeutics, Alexia Dumont Employee of: Domain Therapeutics, Luc Baron Employee of: Domain Therapeutics, Célia Halter Employee of: Domain Therapeutics, Meriem Sémache Employee of: Domain Therapeutics, Arturo Mancini Employee of: Domain Therapeutics, Camille Amalric Employee of: Domain Therapeutics, Marie Giambelluco Employee of: Domain Therapeutics, Nathalie Lenne Employee of: Domain Therapeutics, Marjorie Sidhoum Employee of: Domain Therapeutics, Christel Franchet Employee of: Domain Therapeutics, Stanislas Mayer Employee of: Domain Therapeutics, Xavier Leroy Employee of: Domain Therapeutics, Stephan Schann Employee of: Domain Therapeutics DOI: 10.1136/annrheumdis-2020-eular.5353

\section{OP0237 THROMBOEMBOLIC SAFETY PROFILE OF TOFACITINIB AND BARICITINIB: AN ANALYSIS OF WHO VIGIBASE}

E. Vallejo-Yagüe ${ }^{1}$, S. Weiler ${ }^{1,2}$, A. M. Burden ${ }^{1} .{ }^{1}$ ETH Zürich, Department of Chemistry and Applied Biosciences, Zürich, Switzerland; ${ }^{2}$ National Poisons Information Centre, Tox Info Suisse, Associated Institute of the University of Zurich, Zurich, Switzerland

Background: The Janus Kinase (JAK) inhibitors tofacitinib and baricitinib are new targeted treatments for rheumatoid arthritis. Recent concerns regarding the risk of thrombosis have led to warnings by the European Medicines Agency (EMA) and the Food and Drug Administration (FDA) ${ }^{1,2}$.

Objectives: To examine the safety reporting of tofacitinib and baricitinib, with focus on thromboembolic events.

Methods: Individual case safety reports (ICSRs) for tofacitinib and baricitinib were retrieved from the World Health Organization (WHO) global database (VigiBase) in April 2019. The primary outcomes were deep vein thrombosis (DVT) and pulmonary thrombosis (PT) or pulmonary embolism (PE). A disproportionality analysis was conducted by estimating the reporting odds ratio (ROR) and $95 \%$ confidence intervals (Cls) to compare the observed versus expected reporting ratio of DVT or PTIPE for tofacitinib or baricitinib. The ROR were calculated worldwide and stratifying by reporting from Europe or the US. In a secondary analysis, further thrombotic-related outcomes were investigated.

Results: In both tofacitinib $(n=40,017)$ and baricitinib $(n=2,138)$ ICSRs, patients with reported DVT or PTIPE were older and had higher reporting of pro-thrombotic medications (e.g., contraceptives) or indicators of thromboembolic risk (i.e., antithrombotic treatment). The use of tofacitinib was associated with a significant increased reporting for DVT (ROR: 2.37 95\% Cl 1.23-4.56) and PTIPE (ROR $2.3895 \% \mathrm{Cl} 1.45-3.89$ ) in Europe. In the US, tofacitinib was only associated with an elevated reporting of PT (ROR: $2.05 \% \mathrm{Cl}$ 1.45-2.90). Baricitinib was associated with a 3-fold increased risk of reporting for DVT (ROR: $3.4795 \% \mathrm{Cl}$ 2.18-5.52) or PTIPE (ROR: $3.4495 \% \mathrm{Cl}$ 2.43-4.88) in Europe, which accounted for $97 \%$ of all baricitinib ICSRs. Secondary thrombotic-related outcomes were poorly reported overall in VigiBase.

Conclusion: This study supports the cautious use of JAK inhibitors in patients with rheumatoid arthritis who have a high thrombotic risk profile. Moreover, a potential class effect of JAK inhibitors cannot be ruled out.

\section{References:}

[1] FDA Drug Safety Communication. Safety trial finds risk of blood clots in the lungs and death with higher dose of tofacitinib (Xeljanz, Xeljanz XR) in rheumatoid arthritis patients; FDA to investigate. Food and Drug Administration (FDA) http://www.fda.gov/drugs/drug-safety-and-availability/ safety-trial-finds-risk-blood-clots-lungs-and-death-higher-dose-tofacitinibxeljanz-xeljanz-xr (2019).

[2] EMA confirms Xeljanz to be used with caution in patients at high risk of blood clots. EMA/608520/2019. https://www.ema.europa.eu/en/documents/ referral/xeljanz-article-20-procedure-ema-confirms-xeljanz-be-used-caution-patients-high-risk-blood-clots_en.pdf (2019).

Acknowledgments: We are thankful to every pharmacovigilance centre and contributor to the WHO Programme for International Drug Monitoring and VigiBase.

While the authors used data from the VigiBase, the WHO global database of ICSRs as a source of information, the conclusions do not represent the opinion of the Uppsala Monitoring Centre (UMC) or the WHO.

Disclosure of Interests: Enriqueta Vallejo-Yagüe Employee of: Synovo GmbH 2012-2018 (not related to this abstract), Stefan Weiler Consultant of: Gedeon-Richter for drug safety 2017 (not related to this abstract), Andrea Michelle Burden: None declared DOI: 10.1136/annrheumdis-2020-eular.2823

\begin{tabular}{|l|l}
\hline OP0238 & RISK OF HERPES ZOSTER IN PATIENTS WITH \\
RHEUMATOID ARTHRITIS UNDER BIOLOGICAL, \\
TARGETED SYNTHETIC, AND CONVENTIONAL \\
SYNTHETIC DMARD TREATMENT
\end{tabular}

A. Strangfeld $^{1}$, I. Redeker ${ }^{1}$, J. Kekow ${ }^{2}$, G. R. Burmester ${ }^{3}$, J. Braun ${ }^{4}$, A. Zink ${ }^{1,3}$. ${ }^{1}$ German Rheumatism Research Centre, Berlin, Germany; ${ }^{2}$ Scientific Advisory Board, Vogelsang-Gommern, Germany; ${ }^{3}$ Charité University Medicine, Berlin, Germany; ${ }^{4}$ Rheumatologist, Herne, Germany

Background: The risk of herpes zoster $(\mathrm{HZ})$ is higher in patients with rheumatoid arthritis (RA) than in the general population. This risk is further increased with biologic disease-modifying anti-rheumatic drugs (bDMARDs) such as tumour necrosis factor inhibitors (TNFi) and targeted synthetic (ts)DMARDs such as Janus kinase inhibitors (JAKi) compared to patients taking conventional synthetic (cs)DMARDs such as methotrexate (MTX).

Objectives: To compare incidence rates of $\mathrm{HZ}$ in RA patients under treatment with bDMARDs, tsDMARDs and csDMARDs with different modes of action and to find potential risk factors.

Methods: Data of patients enrolled in the German biologics register RABBIT from 2007 onwards with the start of a bDMARD, tsDMARD or a change in csDMARD treatment were analysed. Patients were included when at least one follow-up documentation was available. All HZ events reported until 30 April 2019 were identified and assigned to treatments administered within the 3 month period prior to the $\mathrm{HZ}$ event. Crude incidence rates (IR) of $\mathrm{HZ}$ were calculated per 1,000 patient years (py). Cox regression was applied to investigate risk factors for the occurrence of $\mathrm{HZ}$ with and without inverse probability weights (IPW) to adjus for confounding by indication.

Results: Data of 12,470 patients $(53,218$ py of observation) were included in the analysis. A total of $452 \mathrm{HZ}$ cases in 433 patients were reported, of which 52 events were serious. The crude IRs per 1,000 py are illustrated by Figure. Adjusted for age, sex, and glucocorticoid use, a significantly increased risk was observed for treatment with monoclonal TNF antibodies (hazard ratio [HR], 1.55 [95\% Cl, 1.21-2.00]), B-cell targeted therapies ( $\mathrm{HR}, 1.45$ [95\% Cl, 1.07-1.97]), and tsDMARDs (HR, 3.55 [95\% Cl, 2.33-5.41]). Treatment with soluble TNF receptors, T-cell co-stimulation modulator, and IL-6 inhibitors were not significantly associated (Table). Adjustment with IPW amplified the effect and treatment with T-cell co-stimulation modulator and IL-6 inhibitors were also significantly associated with a higher risk compared to csDMARD treatment (Table).

Conclusion: This is the first analysis in a European prospective cohort study comparing the incidence rates and risk of $\mathrm{HZ}$ in $\mathrm{RA}$ patients under treatment with six different modes of action within one cohort to cSDMARD treatment. We found a significant association between $\mathrm{HZ}$ and treatment with JAKi. Our results also confirm a higher risk for monoclonal TNF antibodies and show a similar result for the T-cell co-stimulation modulator and B-cell targeted therapies. This study clearly supports systematic $\mathrm{HZ}$ vaccination of RA patients. 